\title{
Modification of sawtooth oscillations with ICRF waves in the JET tokamak
}

\author{
M.J.Mantsinen ${ }^{1,2}$, B. Alper ${ }^{3}$, C. Angioni ${ }^{1}$, R. Buttery ${ }^{3}$, S. Coda ${ }^{4}$, \\ L.-G. Eriksson ${ }^{5}$, J.P. Graves ${ }^{4}$, T. Hellsten ${ }^{6}$, D. Howell ${ }^{3}$, L.C. Ingesson ${ }^{7,8}$, \\ T. Johnson ${ }^{6}$, V. Kiptily ${ }^{3}$, M. Lennholm ${ }^{5}$, M.-L. Mayoral ${ }^{3}$, A. Mück ${ }^{1}$, \\ F. Nabais ${ }^{9}$, F. Nave ${ }^{9}$, J.-M. Noterdaeme ${ }^{1,10}$, J. Ongena ${ }^{11}$, O. Sauter ${ }^{4}$, \\ S.E. Sharapov ${ }^{3}$, E. Westerhof ${ }^{8}$, JET-EFDA Task Force H, JET-EFDA \\ Task Force M, and JET-EFDA Contributors*

\footnotetext{
${ }^{1}$ Max Planck IPP-EURATOM Assoziation, Boltzmann-Str. 2, D-85748 Garching, Germany ${ }^{2}$ Helsinki Univ. of Technology, Association Euratom-Tekes, P.O.Box 4100, FIN-02015 HUT, Finland

${ }^{3}$ EURATOM/UKAEA Fusion Association, Culham Science Centre, Abingdon Oxon OX14 3DB, UK ${ }^{4}$ EURATOM Confédération Suisse, CRPP-EPFL, 1015 Lausanne, Switzerland

${ }^{5}$ Ass. EURATOM-CEA, CEA/DSM/DRFC, CEA-Cadarache, F-13108 St. Paul lez Durance, France

${ }^{6}$ Association EURATOM-VR, Fusion Plasma Physics, EES, KTH, SE-10044 Stockholm, Sweden

${ }^{7}$ EFDA Close Support Unit - Garching, Boltzmannstr. 2, D-85748 Garching, Germany

${ }^{8}$ FOM Institute for Plasma Physics Rijnhuizen, Ass. EURATOM-FOM, Trilateral Euregio Cluster,

Postbus 1207, NL-3430 BE Nieuwegein, The Netherlands

${ }^{9}$ Associação EURATOM/IST, Centro de Fusão Nuclear, Instituto Superior Técnico, Av Rovisco Pais, 1049-001 Lisbon, Portugal

${ }^{10}$ Gent University, EESA Department, B-9000 Gent, Belgium

${ }^{11}$ Association "EURATOM - Belgian State", Laboratory for Plasma Physics, Ecole Royale Militaire, Renaissancelaan 30, B-1000 Brussels, Belgium
} \\ *See Appendix of M.L.Watkins et al., Fusion Energy 2006 (Proc. 21st Int. Conf. Chengdu, 2006) IAEA,
} (2006).

\begin{abstract}
Methods of modifying sawtooth oscillations using waves in the ion cyclotron range of frequencies (ICRF) in the JET tokamak are presented. Examples of sawtooth stabilization by ICRF-accelerated high-energy ions are shown, including experiments with ICRF-acceleration of ${ }^{4} \mathrm{He}$-beam ions to simulate the effects of fusion born alpha particles. With high power ICRF heating in low-density plasmas, fast ion stabilization of sawteeth is lost and a new type of small-period and small-amplitude sawteeth appears. ICRF-induced radial pinch with toroidally asymmetric waves is found to be useful in affecting the radial profile of the ICRF-driven fast ion populations and thereby their influence on sawteeth. Ion cyclotron current drive (ICCD) applied close to the sawtooth inversion radius is effective in modifying the sawtooth period. The latest achievements include the successful application of ICCD to shorten the fast-ioninduced long-period sawteeth and thereby avoid triggering of neoclassical tearing modes (NTMs).
\end{abstract}




\section{INTRODUCTION}

The sawtooth oscillations, caused by the $n=1 / m=1$ internal kink mode, give rise to repetitive redistribution in the core of a tokamak plasma when the central safety factor $\mathrm{q}_{0}$ is below one. Here, $\mathrm{m}$ and $\mathrm{n}$ are the poloidal and toroidal mode numbers, respectively. Therefore, sawteeth are of particular importance for L-mode and H-mode plasmas with $\mathrm{q}_{0}<1$, the latter being one of the baseline scenarios for ITER.

The sawtooth oscillations play a number of key roles in plasmas with $\mathrm{q}_{0}<1$. They are well known to remove impurities from the plasma core, which could find important applications in helium ash removal in a future fusion reactor based on deuterium-tritium fuel. Long period, i.e. stabilized, sawteeth give rise to centrallypeaked density and temperature profiles, which can have a positive effect of increasing the attainable fusion power. High-energy ions, such as $3.5 \mathrm{MeV}$ alpha particles $\left({ }^{4} \mathrm{He}\right.$ ions) born in deuterium-tritium fusion reactions, typically have such a stabilizing effect $[1,2]$. This stabilizing effect is found to be due to fast ions having bounceaveraged precessional drift frequencies $\omega_{\mathrm{D} \text {,fast }}$ in excess of the mode frequency $\omega$ [1]. Unfortunately, a number of drawbacks associated with long-period sawteeth have been discovered. These include destabilization of neoclassical tearing modes (NTMs) by sawtooth crashes following long-period sawteeth [3]. Unless these modes can be avoided or stabilized, they could significantly limit the attainable fusion performance as well as cause disruptions. One of the ways to achieve this is to shorten the sawtooth period, i.e. destabilize the sawteeth, so that seed islands capable of triggering NTMs do not appear in the first place. Thus, there are reasons for both lengthening and shortening of the sawtooth period and means for effective sawtooth control is called for. Possible techniques include modifications via the plasma current and/or conductivity profiles to modify the magnetic shear near $\mathrm{q}=1$ where the kink mode is located, or via fast ions. For all of these, ICRF waves provide a suitable tool since they can provide localized current drive and heating as well as accelerate resonant ions to high energies.

The present paper reviews the achievements in modifying sawtooth oscillations using ICRF waves on the JET tokamak $\left(\mathrm{R}_{0} \approx 3 \mathrm{~m}, \mathrm{a} \approx 1 \mathrm{~m}\right)$. Due to the wide frequency range of launched waves $(23-57 \mathrm{MHz})$ and the phasing capability of the JET ICRF antennas [4], several ICRF heating and current drive schemes are possible for sawtooth control. Furthermore, ICRF-accelerated resonant ions in JET often have energies up in the $\mathrm{MeV}$ range. Due to the relatively large plasma size and current, these ions are typically well-confined and can be used to simulate to some extent the effects of fusion-born alpha-particles. With phased antennas, toroidally directed ICRF waves can also be launched to stabilize or destabilize sawteeth by current drive in the vicinity of $\mathrm{q}=1$. In the following, results on modification of sawteeth via fast ions, via current drive and via their combination using ICRF waves on JET are presented.

\section{MODIFICATIONS OF SAWTEETH VIA ICRF FAST IONS}

The first observations of the stabilizing influence of ICRF-accelerated fast ions on the 
sawtooth instability were made in 1980's following an increase in the coupled ICRF power [5]. Sawtooth periods of up to $5 \mathrm{~s}$ where obtained in plasmas with on-axis safety factor below one (Fig. 1). More recently, third harmonic ion cyclotron resonance heating of ${ }^{4} \mathrm{He}$ beam ions has been used to produce high-energy populations of ${ }^{4} \mathrm{He}$ ions to simulate $3.5 \mathrm{MeV}$ fusion-born alpha particles and their effects [6]. Strongest fast ${ }^{4} \mathrm{He}$ populations have been obtained with highest $(120 \mathrm{keV})$ energy ${ }^{4} \mathrm{He}$ beam seed ions, as expected. Concomitant sawtooth stabilization was observed, with sawtooth period increasing with the fast ion energy content. Clear degradation of the plasma temperature and energy content was also observed due to NTMs, triggered at sawtooth crashes following long period sawteeth (Fig. 2) [6,7].

For sawtooth stabilization by fast ions the fast ion pressure inside the $q=1$ surface is a key quantity. The available techniques to tailor the ICRF-driven fast ion pressure inside the $\mathrm{q}=1$ surface includes varying ICRF power, resonant ion concentration and resonance position, as well as using multiple frequencies or ICRFinduced radial pinch with toroidally directed waves. All of these methods have been shown to modify sawteeth on JET. In the following, we discuss in more detail the effects of the ICRF-induced pinch of resonating ions which arises as an ion interacting with a wave receives a change in toroidal momentum [8]. For a trapped particle, this leads to the radial transport of banana tips either inwards or outwards depending on the toroidal direction of the wave. For the inward pinch with waves in the co-current direction, the fast ion orbit is eventually detrapped into co-passing orbits that reside on the low field side of the resonance $\omega=n \omega_{\text {ci }}$ [9]. Experimental evidence of this effect has been obtained on the JET tokamak with high-power $\mathrm{H}$ and ${ }^{3} \mathrm{He}$ minority heating $[10,11]$. In particular, with a central resonance and inward pinch using co-current propagating waves, longer sawtooth periods have been observed (Fig. 3), consistent with a larger fast ion pressure in the centre [10].

Experimentally, sawtooth stabilization by ICRF-accelerated fast ions has been found to fail in plasmas with low density, $\mathrm{n}_{\mathrm{e}}<\mathrm{n}_{\mathrm{e}, \text { crit }} \approx 2 \times 10^{19} \mathrm{~m}^{-3}$, and high-power ICRF heating, producing fast ions with a total energy content comparable to that of thermal plasma [12]. Instead, short period and small-amplitude 'grassy' sawteeth appear (Fig. 4), accompanied by $\mathrm{n}=1$ fishbone-like modes and Alfvén eigemodes. Possible explanations for the loss of fast ion stabilizing effect have been searched for: The orbit width of the fast ions exceeding the $\mathrm{q}=1$ radius [13] and possible generation of supra-thermal electrons during sawtooth crashes similar to [14] have been put forward as the main density-dependent effects [15]. The effect of fast ion profiles on sawteeth has been studied with on-axis and off-axis ICRF heating and with ICRF waves launched predominantly in the co-current and counter-current direction [15]. In all cases 'grassy' sawteeth were obtained in spite of the different fast ion radial profiles and average energies. However, the sharp transition to grassy sawteeth was found to mainly correlate with an increase in the intensity of fast electron bremsstrahlung (FEB) emission from the central region of the plasma, measured in the energy range from 150 to $450 \mathrm{keV}$, just before the transition to grassy sawteeth [15]. Bursts of supra-thermal ECE emission have also been seen during the sawtooth crashes. Estimates show that in these low-density high-temperature discharges the 
electric field is close to the critical field for the runaway electrons, $E \approx E_{\text {crit }}$, so that sawtooth crashes could affect the runaways by generating electric field comparable to the near-threshold value $E_{\text {crit }}-E$ [15]. The observations suggest that supra-thermal electrons may carry a significant current in the vicinity of $\mathrm{q}=1$ and thus play a role in sawtooth dynamics.

\section{MODIFICATIONS VIA ION CYCLOTRON CURRENT DRIVE}

Due to their average finite wave number $\mathrm{k}_{\|}$, toroidally directed ICRF waves couple asymmetrically to ions and electrons in the velocity space and can drive current. The method of driving current by heating either minority ions with toroidally directed waves at a frequency equal to the ion cyclotron frequency, or majority ions at harmonics of the cyclotron frequency, was originally proposed by Fisch [16]. Because of the Doppler shift of the cyclotron resonance, $\omega=n \omega_{c i}+\mathrm{k}_{\|} \mathrm{v}_{\|}$, directed waves interact resonantly with either co-going or counter-going ions, depending on which side of the cyclotron resonance the interaction takes place and on the direction of the wave propagation. As a result, there is an effective transfer of ions either to co-passing or counter-passing orbits, and thus a driven current. For passing ion current, it is beneficial to have rather strong pitch angle scattering by keeping the average fast ion energy comparable to the critical energy [17]. Current drive, even with toroidally symmetric spectra, is possible by trapped ions due to their finite orbit widths and due to effects of non-standard orbits [9]. The trapped ion current has a dipolar shape and for typical JET parameters can exceed the current drive by passing ions [18].

While the net driven current is typically rather small, the local effect of ICCD on the magnetic shear can be rather strong. This has been clearly demonstrated in experiments where ion cyclotron current drive has been used to modify the magnetic shear near $\mathrm{q}=1$ and thereby affect the sawtooth activity (Fig. 5). The sawtooth period has been varied in ICRF-only discharges by more than one order of magnitude due to changes in the magnetic shear near $q=1$, using hydrogen minority $\left(\omega \approx \omega_{\mathrm{cH}}\right)$ ICCD [18-21]. Experiments have also been carried out with directed ICRF waves tuned to the second harmonic hydrogen resonance $\left(\omega \approx 2 \omega_{\mathrm{cH}}\right)$ to affect the sawtooth period and amplitude in discharges with ICRF only and in discharges with combined ICRF and NBI [3, 22-24]. In these experiments the $\mathrm{m}=3, \mathrm{n}=2$ NTM seed island has been controlled by controlling the sawtooth behaviour. By adding off-axis ICCD to destabilize sawteeth, larger beta values obtained without triggering NTMs than with ICCD applied to stabilize sawteeth (Fig. 6).

\section{COMBINED SCHEMES}

In ITER, fusion-born alpha particles are expected to stabilize sawteeth and crashes of alpha-stabilized long-period sawteeth may give rise to seed islands capable of triggering NTMs. It is therefore of importance to study ways in which fast-ion stabilized long-period sawteeth can be shortened. Experimental evidence for the ability of localized current drive to destabilize fast-ion-induced long sawtooth periods has 
been obtained on JET using ICRF waves. In these experiments ICRF waves have performed two tasks: (1) they provide fast ions to stabilize sawteeth and (2) they provide localised current drive at $\mathrm{q}=1$ to influence the magnetic shear and thereby shorten the fast-ion-induced long-period sawteeth [18,25]. Figure 7 shows an overview of such a discharge. Firstly, $3 \mathrm{MW}$ of ICRF power was launched with $+90^{\circ}$ phasing and a hydrogen minority cyclotron resonance close to the magnetic axis to establish fast-ion-induced long sawteeth. Thereafter, ICRF power with $-90^{\circ}$ phasing was applied for ICCD with a hydrogen minority cyclotron resonance close to the $q=1$ surface on the high field side. A clear destabilization of the sawteeth occurred a short period after ICRF power for ICCD was turned on. More recently, these experiments have been extended into more challenging conditions with higher normalized plasma beta to demonstrate the avoidance of NTMs using ICCD control of fast-ion-induced long sawteeth [26].

\section{REFERENCES}

1 F. Porcelli, Plasma Phys. Control. Fusion 33 (1991) 1601.

2 F. Porcelli, D. Boucher, M.N. Rosenbluth, Plasma Phys. Control. Fusion 38 (1996) 2163.

3 O. Sauter et al., Phys. Rev. Lett. 88 (2002) 105001.

4 A. Kaye et al., Fusion Eng. Design 24 (1994) 1.

D.J. Campbell et al., Phys. Rev. Lett. 60 (1988) 2148.

M.J. Mantsinen et al., Phys. Rev. Lett. 88 (2002) 105002.

J. Pamela et al., Nucl. Fusion 42 (2002) 1014.

8 L. Chen, J. Vaclavik, and G. Hammett, Nucl. Fusion 28 (1988) 389.

9 T. Hellsten, J. Carlsson, L.-G. Eriksson, Phys. Rev. Lett. 74 (1995) 3612.

10 L.-G. Eriksson, et al. Phys. Rev. Lett. 81 (1998) 1231.

11 M.J. Mantsinen et al., Phys. Rev. Lett. 89 (2002) 115004.

12 M.J. Mantsinen et al., Plasma Phys. Control. Fusion 42 (2001) 1291.

13 F. Nabais et al., Proc. 20th Int. Conf. on Fusion Energy 2004 (Vilamoura, 2004) (Vienna: IAEA) paper $\mathrm{TH} / 5-3$.

14 P.V. Savrukhin, Phys. Rev. Lett. 68 (2001) 3036.

15 S. E. Sharapov et al., Nucl. Fusion 45 (2005) 1168.

16 N. Fisch, Nucl. Fusion 21 (1981) 15.

17 T.H. Stix, Nucl. Fusion 15 (1975) 737.

18 L.-G. Eriksson et al., Nucl. Fusion 46 (2006) S951.

19 D.F.H. Start et al., Proc. International Conf. on Plasma Physics (Innsbruck, 1992) vol 16C (part II) (Geneva: European Physical Society 1992) p 897.

20 V.P. Bhatnagar et al., Nucl. Fusion 34 (1994) 1579.

21 M.-L. Mayoral et al., Phys. Plasmas 11 (2004) 2607.

22 M.-L. Mayoral et al., Radio frequency power in plasmas, 14th Topical Conf. (Oxnard, California 2001) (New York: Americal Institute of Physics) AIP Conf. Proc. 595 (2001) 106.

23 E. Westerhof et al., Nucl. Fusion 42 (2002) 1324.

24 M.J. Mantsinen et al., Plasma Phys. Control. Fusion 44 (2002) 1521.

25 L.-G. Eriksson et al., Phys. Rev. Lett. 92 (2004) 235004.

26 S. Coda et al., 'NTM prevention by ICCD control of fast-ion-stabilized sawteeth', 34th European Physical Society Conference on Plasma Physics, Warsaw, Poland (2007). 


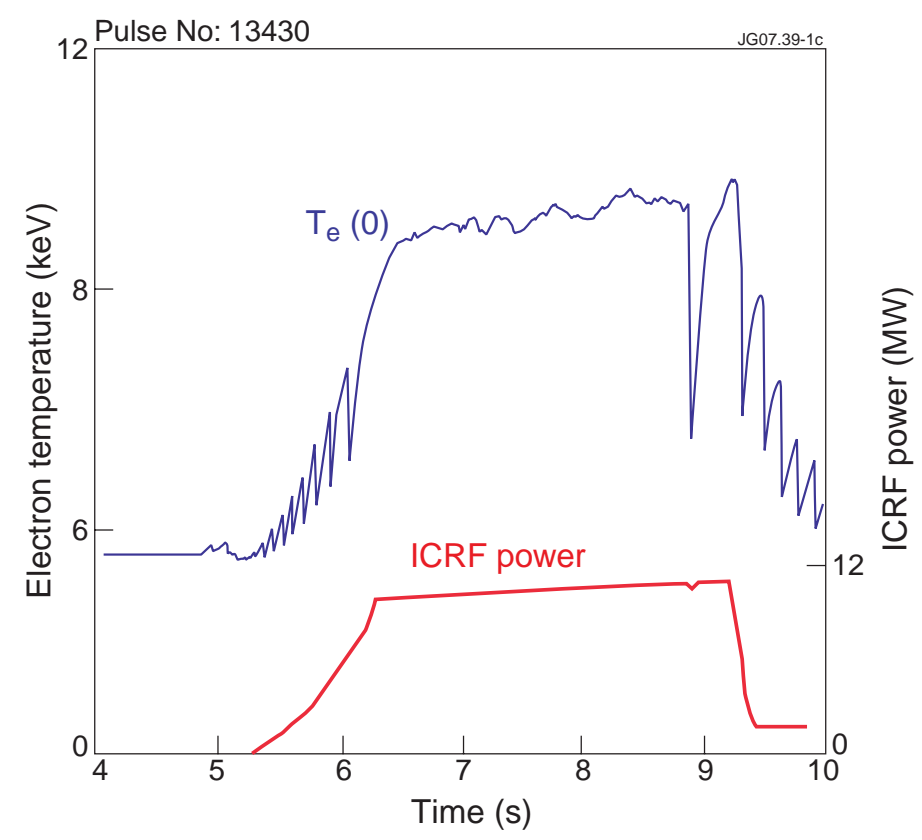

FIGURE 1 Central electron temperature and ICRF power for discharge 13430 with sawtooth stabilization due to ICRF-accelerated fast ions. Here, a sawtooth period of up to $3.5 \mathrm{~s}$ is measured.

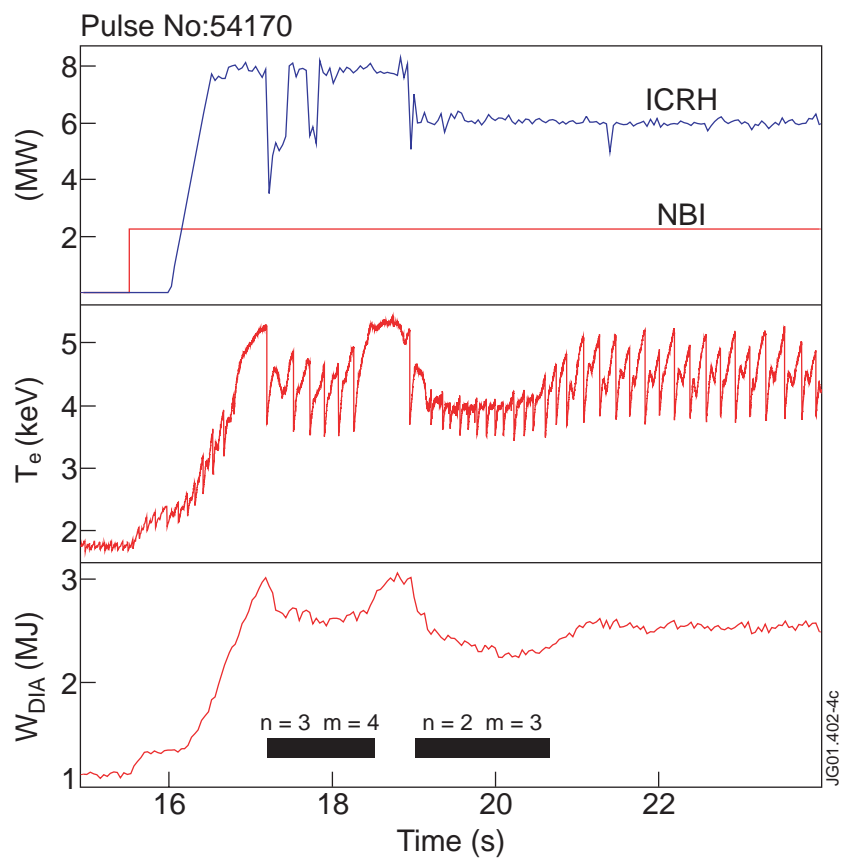

FIGURE 2 ICRF and NBI power, electron temperature and diamagnetic plasma energy for discharge 54170 with $\omega \approx 3 \omega\left({ }^{4} \mathrm{He}\right)$ and $120 \mathrm{keV}$ deuterium NBI. Also shown are the time intervals where NTMs with $n=3 / m=4$ and $n=2 / m=3$, triggered by the crashes of long-period sawteeth, are observed. 


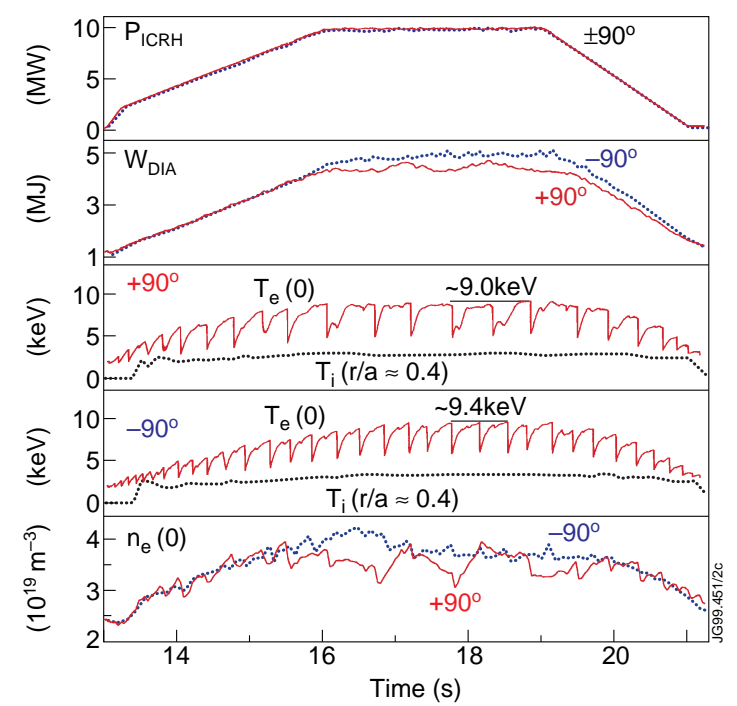

FIGURE 3 ICRF power, diamagnetic plasma energy, central electron temperature, ion temperature at $\mathrm{r} / \mathrm{a}=0.4$ and central electron density for two discharges with different ICRF phasings (discharges 41514 and 41515).
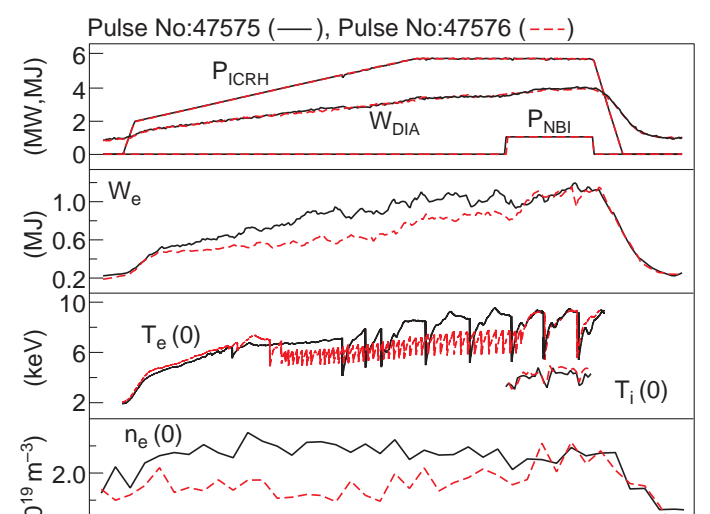

$\stackrel{\circ}{=} 1.0$

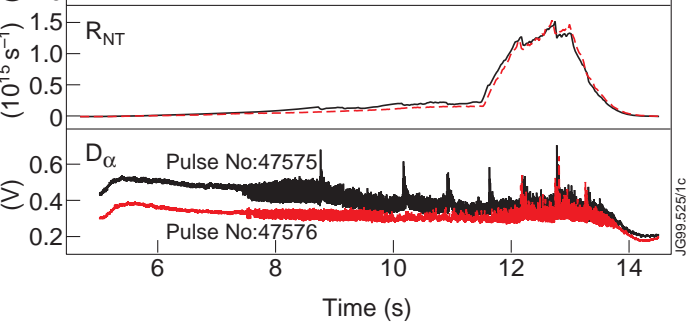

FIGURE 4 ICRF and NBI power, diamagnetic plasma energy, electron energy, central electron and ion temperatures, central electron density, deuterium-deuterium neutron rate and $\mathrm{D}_{\alpha}$ signal for two discharges with differing plasma densities.

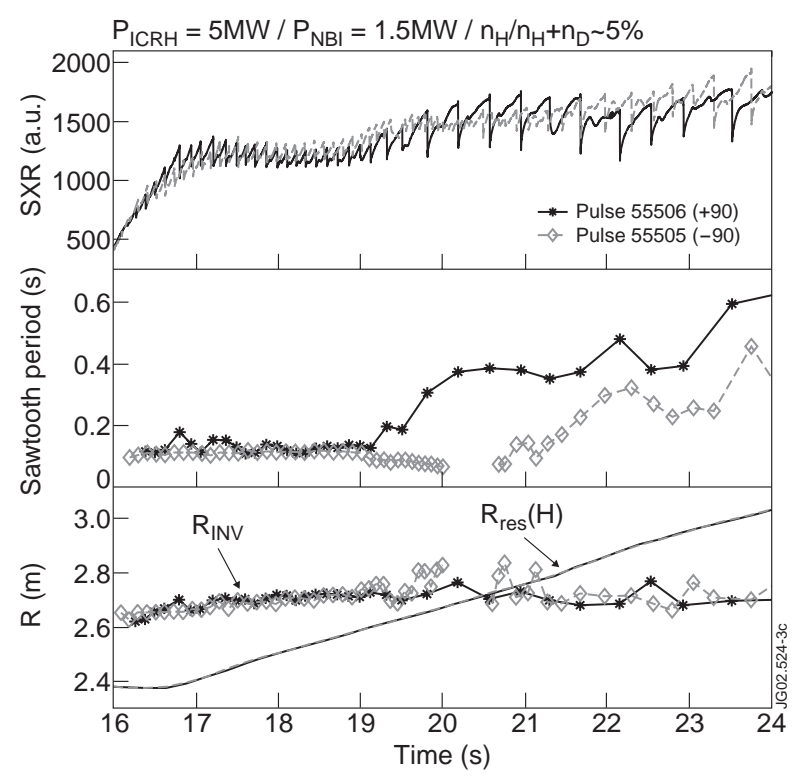

FIGURE 5 Soft x-ray emission, sawtooth period, sawtooth inversion radius $R_{\text {inv }}$ and first harmonic hydrogen cyclotron resonance layer $R_{\text {res }}(\mathrm{H})$ for two discharges with different ICRF phasings. The magnetic field and the plasma current were ramped from $2.2 \mathrm{~T} / 2.2 \mathrm{MA}$ at $t=17 \mathrm{~s}$ to $2.75 \mathrm{~T} / 2.75 \mathrm{MA}$ at $\mathrm{t}=24 \mathrm{~s}$, respectively. 


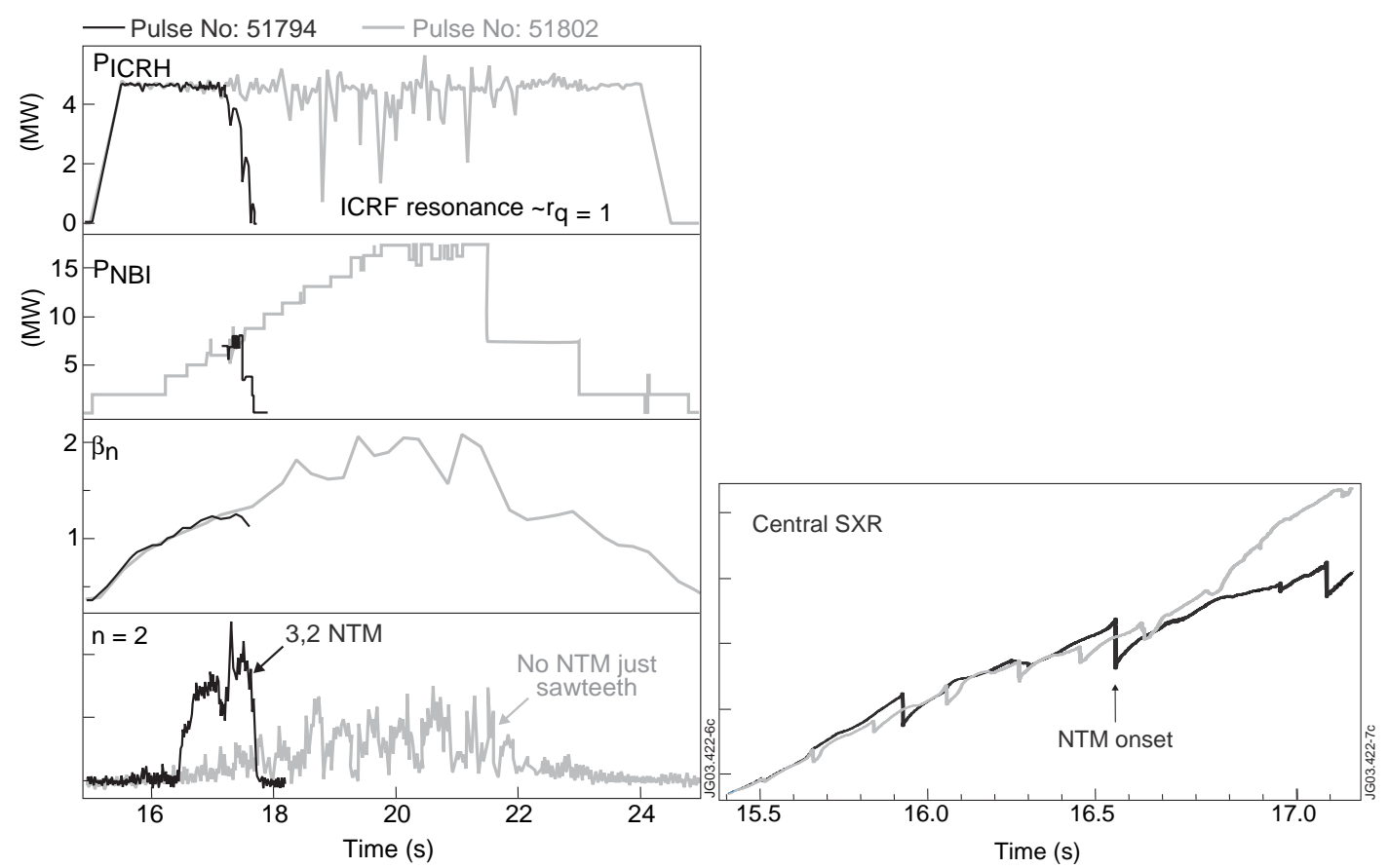

FIGURE 6 Comparison of two discharges with ICCD applied with resonance close to the $q=1$ surface but with different ICRF phasing.

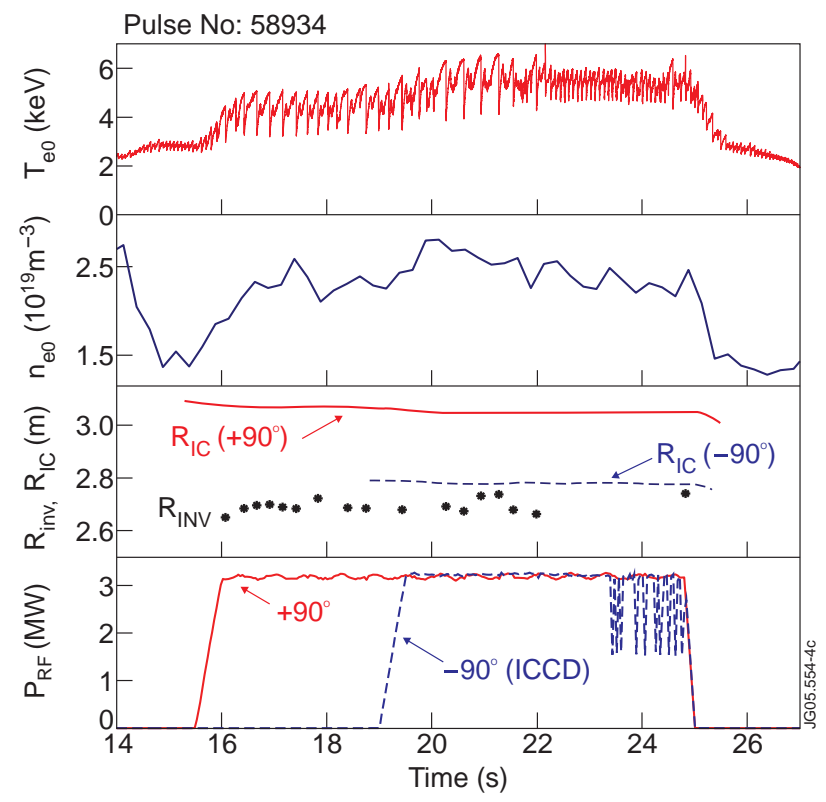

FIGURE 7 Overview of a discharge demonstrating shortening of fast-ion-induced long sawtooth periods by minority ICCD. The traces from the bottom are ICRF power for $+90^{\circ}$ central $\mathrm{H}$ resonance, $-90^{\circ}$ power for minority ICCD with a $\mathrm{H}$ resonance on the high field side; major radii $\left(\mathrm{R}_{\mathrm{IC}}\right)$ of the cyclotron resonances, major radius of the sawtooth inversion radius $\left(\mathrm{R}_{\mathrm{inv}}\right)$, central electron density and central electron temperature. 